\title{
Probing the Relationship Between Interfacial Concentrations and Lipase Activity in Cationic W/O Microemulsions: A Quantitative Study by Chemical Trapping
}

\author{
Antara Dasgupta, Debapratim Das, and Prasanta Kumar Das*广 \\ Department of Biological Chemistry, Indian Association for the Cultivation of Science, \\ Jadavpur, Kolkata - 700032, India.
}

Supporting Information 
Table S1: Calibration plots of 1-ArOH and 1-ArBr with mobile phase of 75/25 acetonitrile/water (used for short chain 7 and 11).

\begin{tabular}{cccc}
\hline & & \multicolumn{3}{c}{ Peak areas } \\
\cline { 3 - 4 }$[1-\mathrm{ArOH}](\mathrm{M})$ & {$[1-\mathrm{ArBr}](\mathrm{M})$} & $\begin{array}{c}1-\mathrm{ArOH} \\
(\mu \mathrm{V})\end{array}$ & $\begin{array}{c}1-\mathrm{ArBr} \\
(\mu \mathrm{V})\end{array}$ \\
\hline $1.5 \times 10^{-5}$ & $1.5 \times 10^{-5}$ & 131529 & 156789 \\
$1.2 \times 10^{-4}$ & $6 \times 10^{-5}$ & 911647 & 748520 \\
$2.4 \times 10^{-4}$ & $1.2 \times 10^{-4}$ & 1569915 & 1483930 \\
$2.85 \times 10^{-4}$ & $1.8 \times 10^{-4}$ & 1721768 & 2525739
\end{tabular}

HPLC calibration curve for 1-ArOH: Area in $\mu \mathrm{V}=8.56 \times 10^{9}$ [1-ArOH] M -34985(5points correlation coefficient $=0.99)$. HPLC calibration curve for $1-\mathrm{ArBr}$ : Area in $\mu \mathrm{V}=1.36 \times 10^{10}$ [1-ArBr]M - 65016 (5 points correlation coefficient =0.99).

Table S2: Calibration plots of 1-ArOH and 1-ArBr with mobile phase of 70/30 acetonitrile/water (used for short chain 8-10).

\begin{tabular}{cccc}
\hline & & \multicolumn{3}{c}{ Peak areas } \\
\cline { 3 - 4 }$[1-\mathrm{ArOH}](\mathrm{M})$ & {$[1-\mathrm{ArBr}](\mathrm{M})$} & $\begin{array}{c}1-\mathrm{ArOH} \\
(\mu \mathrm{V})\end{array}$ & $\begin{array}{c}1-\mathrm{ArBr} \\
(\mu \mathrm{V})\end{array}$ \\
\hline $1.52 \times 10^{-5}$ & $1.65 \times 10^{-5}$ & 141345 & 94554 \\
$1.22 \times 10^{-4}$ & $6.6 \times 10^{-5}$ & 1087602 & 407462 \\
$2.83 \times 10^{-4}$ & $1.32 \times 10^{-4}$ & 1605097 & 814386 \\
$2.89 \times 10^{-4}$ & $1.98 \times 10^{-4}$ & 2083860 & 1216973
\end{tabular}

HPLC calibration curve for 1-ArOH: Area in $\mu \mathrm{V}=8.0 \times 10^{9}$ [1-ArOH] $\mathrm{M}+28013$ (5 points correlation coefficient $=0.99)$. HPLC calibration curve for 1 -ArBr: Area in $\mu \mathrm{V}=6.0 \times 10^{9}[1$ $\mathrm{ArBr}] \mathrm{M}$ - 2741 (5 points correlation coefficient =1). 
Table S3: Calibration plots of 1-ArOH and 1-ArBr with mobile phase of 70/30 acetonitrile/water (used for short chain 12).

\begin{tabular}{|c|c|c|c|}
\hline \multirow[b]{2}{*}[1-\mathrm{ArOH}]{$(\mathrm{M})$} & \multirow[b]{2}{*}[1-\mathrm{ArBr}]{$(\mathrm{M})$} & \multicolumn{2}{|c|}{ Peak areas } \\
\hline & & $\begin{array}{c}\text { 1-ArOH } \\
(\mu \mathrm{V})\end{array}$ & $\begin{array}{c}1-\mathrm{ArBr} \\
(\mu \mathrm{V})\end{array}$ \\
\hline $1.52 \times 10^{-5}$ & $1.65 \times 10^{-5}$ & 137319 & 109694 \\
\hline $1.22 \times 10^{-4}$ & $6.6 \times 10^{-5}$ & 1125525 & 415757 \\
\hline $1.83 \times 10^{-4}$ & $1.32 \times 10^{-4}$ & 1678523 & 866684 \\
\hline $2.44 \times 10^{-4}$ & $1.98 \times 10^{-4}$ & 2140629 & 1244954 \\
\hline $2.89 \times 10^{-4}$ & $3.13 \times 10^{-4}$ & 2412182 & 1970594 \\
\hline
\end{tabular}

HPLC calibration curve for 1-ArOH: Area in $\mu \mathrm{V}=9.0 \times 10^{9}[1-\mathrm{ArOH}] \mathrm{M}+35322(5$ points correlation coefficient $=0.99)$. HPLC calibration curve for 1 -ArBr: Area in $\mu \mathrm{V}=6.0 \times 10^{9}[1$ $\mathrm{ArBr}] \mathrm{M}+7268$ (5 points correlation coefficient $=0.99)$.

Table S4: Calibration plots of 1-ArOH and 1-ArOBu with mobile phase of 60/40 upto 3.5 min and then $80 / 20$ of acetonitrile /water.

\begin{tabular}{cccc}
{$[1-\mathrm{ArOH}](\mathrm{M})$} & {$[1-\mathrm{ArOBu}](\mathrm{M})$} & $\begin{array}{c}1-\mathrm{ArOH} \\
(\mu \mathrm{V})\end{array}$ & $\begin{array}{c}1-\mathrm{ArOBu} \\
(\mu \mathrm{V})\end{array}$ \\
\cline { 3 - 4 } $4.5 \times 10^{-4}$ & $2.0 \times 10^{-6}$ & 29496209 & 678800 \\
$5 \times 10^{-4}$ & $2.9 \times 10^{-6}$ & 34137261 & 815863 \\
$5.5 \times 10^{-4}$ & $4.0 \times 10^{-6}$ & 35718094 & 1234387 \\
$5.9 \times 10^{-4}$ & $5.0 \times 10^{-6}$ & 37893611 & 1470157 \\
$6.5 \times 10^{-4}$ & $6.0 \times 10^{-6}$ & 43383120 & 1973587
\end{tabular}

HPLC calibration curve for 1-ArOH: Area in $\mu \mathrm{V}=7.0 \times 10^{10}[1-\mathrm{ArOH}] \mathrm{M}+47593$ (5 points correlation coefficient $=0.97)$. HPLC calibration curve for 1 -AOBu: Area in $\mu \mathrm{V}=3 \times 10^{11}[1$ $\mathrm{ArOBu}] \mathrm{M}-16905$ (5 points correlation coefficient = 0.97). 
Table S5: Calibration plots of 16-ArOH, 16-ArBr and 16-ArOHex with mobile phase of 72/28 methanol/isopropanol.

\begin{tabular}{|c|c|c|c|c|c|}
\hline \multirow{2}{*}{$\begin{array}{c}{[16-\mathrm{ArOH}]} \\
(\mathrm{M})\end{array}$} & \multirow{2}{*}{$\begin{array}{c}{[16-\mathrm{ArBr}]} \\
(\mathrm{M})\end{array}$} & \multirow{2}{*}{$\begin{array}{c}{[16-\mathrm{ArOHex}]} \\
(\mathrm{M})\end{array}$} & \multicolumn{3}{|c|}{ Peak areas } \\
\hline & & & $\begin{array}{c}\text { 16-ArOH } \\
(\mu \mathrm{V})\end{array}$ & $\begin{array}{c}\text { 16-ArBr } \\
(\mu \mathrm{V})\end{array}$ & $\begin{array}{c}\text { 16-ArOHex } \\
(\mu \mathrm{V})\end{array}$ \\
\hline $2.48 \times 10^{-5}$ & $3.48 \times 10^{-5}$ & $5.01 \times 10^{-6}$ & 584648 & 1214235 & 128546 \\
\hline $3.48 \times 10^{-5}$ & $4.5 \times 10^{-5}$ & $7.98 \times 10^{-6}$ & 932602 & 1770791 & 266733 \\
\hline $4.46 \times 10^{-5}$ & $5.52 \times 10^{-5}$ & $1.2 \times 10^{-5}$ & 1205335 & 2139607 & 355965 \\
\hline $5.46 \times 10^{-5}$ & $6.54 \times 10^{-5}$ & $1.59 \times 10^{-5}$ & 1503073 & 2515931 & 556648 \\
\hline $6.44 \times 10^{-5}$ & $7.02 \times 10^{-5}$ & $2.0 \times 10^{-5}$ & 1721353 & 2846314 & 684610 \\
\hline \multicolumn{6}{|c|}{$\begin{array}{l}\text { HPLC calibration curve for } 16-\mathrm{ArOH} \text { : Area in } \mu \mathrm{V}=3.0 \times 10^{10}[16-\mathrm{ArOH}] \mathrm{M}-28188(5 \\
\text { points correlation coefficient }=0.99) \text {. HPLC calibration curve for } 16-\mathrm{ArBr} \text { : Area in } \mu \mathrm{V}= \\
4.0 \times 10^{10}[16-\mathrm{ArBr}] \mathrm{M}-57195(5 \text { points correlation coefficient }=0.99) \text {. HPLC calibration } \\
\text { curve for } 16 \text {-ArOHex: Area in } \mu \mathrm{V}=3.0 \times 10^{10}[16 \text {-ArOHex }] \mathrm{M}-19630 \text { ( } 5 \text { points correlation } \\
\text { coefficient }=0.99) \text {. }\end{array}$} \\
\hline
\end{tabular}


Table S6: HPLC peak areas, observed and normalized product yields for room temperature dediazoniation of $1-\mathrm{ArN}_{2}{ }^{+}$in aqueous solutions of $\mathrm{n}-\mathrm{BuOH}$ at $\mathrm{pH}=6.0$.

\begin{tabular}{|c|c|c|c|c|c|c|c|c|c|c|}
\hline \multirow{2}{*}{$\begin{array}{l}\text { Reaction }^{\mathrm{a}} \\
{\left[1-\mathrm{ArN}_{2}^{+}\right]} \\
\times 10^{-3} \mathrm{M}\end{array}$} & \multirow{2}{*}{$\begin{array}{c}{[n-} \\
\mathrm{BuOH}] \\
(\mathrm{M})\end{array}$} & \multirow{2}{*}{$\begin{array}{c}{\left[\mathrm{H}_{2} \mathrm{O}\right]} \\
(\mathrm{M})\end{array}$} & \multicolumn{2}{|c|}{ Peak areas } & \multicolumn{3}{|c|}{ Observed yields ${ }^{\mathrm{b}}$} & \multicolumn{2}{|c|}{ Normalized yields ${ }^{c}$} & \multirow{2}{*}{$\mathrm{S}_{W}{ }^{\mathrm{A}}$} \\
\hline & & & $\begin{array}{c}1-\mathrm{ArOH} \\
\mu \mathrm{V}\end{array}$ & $\begin{array}{c}\text { 1-ArOBu } \\
\mu \mathrm{V}\end{array}$ & $\begin{array}{c}\text { 1-ArOH } \\
(\%)\end{array}$ & $\begin{array}{c}\text { 1-ArOBu } \\
(\%)\end{array}$ & $\begin{array}{l}\text { total } \\
(\%)\end{array}$ & $\begin{array}{c}1-\mathrm{ArOH} \\
(\%)\end{array}$ & $\begin{array}{c}1-\mathrm{ArOBu} \\
(\%)\end{array}$ & \\
\hline 1.0 & 0.3 & 55.5 & 18956008 & 198016 & 93.1 & 0.25 & 93.3 & 99.7 & 0.3 & 0.55 \\
\hline 1.0 & 0.4 & 53.6 & 18408563 & 273284 & 90.3 & 0.33 & 90.6 & 99.6 & 0.4 & 0.54 \\
\hline 1.0 & 0.5 & 52.9 & 18378064 & 364973 & 90.3 & 0.44 & 90.7 & 99.5 & 0.5 & 0.53 \\
\hline 1.06 & 0.6 & 53.6 & 18018574 & 444109 & 89.6 & 0.53 & 90.1 & 99.4 & 0.6 & 0.54 \\
\hline 1.0 & 0.7 & 53.5 & 18277010 & 486265 & 93.1 & 0.58 & 93.7 & 99.3 & 0.7 & 0.54 \\
\hline 1.0 & 0.8 & 52.7 & 19631988 & 586251 & 96.5 & 0.69 & 97.2 & 99.3 & 0.7 & 0.53 \\
\hline 1.0 & 0.9 & 52.6 & 18669435 & 873635 & 93.1 & 1.02 & 94.1 & 98.9 & 1.1 & 0.53 \\
\hline
\end{tabular}

${ }^{a}$ Reaction mixtures were 3.333 times diluted before injecting into HPLC. A $200 \mu \mathrm{L}$ sample loop was used for injecting the diluted mixtures to bring the peak areas of both $1-\mathrm{ArOH}$ and 1-ArOBu within the peak area range of the calibration graphs. The mobile phase used for separation of products is $60 / 40$ - acetonitrile/water for the fist $3.5 \mathrm{mins}$ at $0.9 \mathrm{ml} / \mathrm{min}$ and then $80 / 20$ - acetonitrile water at $0.4 \mathrm{ml} / \mathrm{min}$ upto 5.0 min and then again at $0.9 \mathrm{ml} / \mathrm{min}$ for the remaining $20 \mathrm{mins}$.

${ }^{\mathrm{b}} \mathrm{HPLC}$ calibration curve for 1-ArOH: Area in $\mu \mathrm{V}=7.0 \times 10^{10}$ [1-ArOH] M+47593(5 points correlation coefficient $\left.=0.99\right)$; $\%$ yield of $1-\mathrm{ArOH}$ $=100 \times[1-\mathrm{ArOH}] \mathrm{M} /\left[1-\mathrm{ArN}_{2}^{+}\right] \mathrm{M}$.

HPLC calibration curve for 1 -ArOBu: Area in $\mu \mathrm{V}=3 \times 10^{11}[1-\mathrm{ArOBu}] \mathrm{M}-16905$ (5 points correlation coefficient $=0.98$ ); $\%$ yield of 1 $\mathrm{ArOBu}=100 \times[1-\mathrm{ArOBu}] \mathrm{M} /\left[1-\mathrm{ArN}_{2}{ }^{+}\right] \mathrm{M}$. Observed yields are reproducible within $\pm 1 \%$ in duplicate experiments.

${ }^{\mathrm{c}}$ Because of quantitative conversions with no significant extraneous peak, observed yields were normalized.

The obtained correlation between $[\mathrm{BuOH}]$ and $\% 1-\mathrm{ArOBu}$ is:

$\% 1-\mathrm{ArOBu}=1.214[\mathrm{BuOH}]-0.1 \quad$ (correlation coefficient $=0.99)$. 
Table S7: HPLC peak areas, observed and normalized product yields for room temperature dediazoniation of $1.0 \mathrm{mM} 1$-ArN $_{2}^{+}$in aqueous $^{-}$ solutions of $7^{\mathrm{a}}$ at $\mathrm{pH}=6.0$.

\begin{tabular}{|c|c|c|c|c|c|c|c|c|c|}
\hline \multirow{2}{*}{$\begin{array}{l}{[7]} \\
(\mathrm{M})\end{array}$} & \multicolumn{2}{|c|}{ Peak areas } & \multicolumn{3}{|c|}{ Observed yields ${ }^{\mathrm{b}}$} & \multicolumn{2}{|c|}{ Normalized yields ${ }^{c}$} & \multirow{2}{*}{$\begin{array}{c}{\left[\mathrm{H}_{2} \mathrm{O}\right]} \\
(\mathrm{M})\end{array}$} & \multirow{2}{*}{$\mathrm{S}_{\mathrm{W}}{ }^{\mathrm{Br}}$} \\
\hline & $\begin{array}{c}1-\mathrm{ArOH} \\
\mu \mathrm{V}\end{array}$ & $\begin{array}{c}1-\mathrm{ArBr} \\
\mu \mathrm{V}\end{array}$ & $\begin{array}{c}1-\mathrm{ArOH} \\
(\%)\end{array}$ & $\begin{array}{c}1-\mathrm{ArBr} \\
(\%)\end{array}$ & $\begin{array}{l}\text { total } \\
(\%)\end{array}$ & $\begin{array}{c}\text { 1-ArOH } \\
(\%)\end{array}$ & $\begin{array}{c}1-\mathrm{ArBr} \\
(\%)\end{array}$ & & \\
\hline 0.1 & 1854237 & 60871 & 96.0 & 3.9 & 99.9 & 96.1 & 3.9 & 55.2 & 22.4 \\
\hline 0.5 & 2284015 & 465144 & 89.4 & 12.5 & 101.9 & 87.7 & 12.3 & 52.7 & 14.8 \\
\hline 1.0 & 2106227 & 756965 & 82.5 & 19.5 & 102.0 & 82.9 & 19.1 & 49.6 & 11.7 \\
\hline 1.5 & 1916001 & 1040166 & 75.9 & 26.1 & 102.0 & 74.4 & 25.6 & 46.6 & 10.7 \\
\hline 2.0 & 1763283 & 1240368 & 69.3 & 30.8 & 100.1 & 69.3 & 30.7 & 43.3 & 9.6 \\
\hline 2.5 & 1727566 & 1526097 & 66.6 & 37.6 & 104.2 & 64.3 & 35.7 & 40.2 & 8.9 \\
\hline 3.0 & 1453054 & 1585341 & 57.4 & 38.9 & 96.3 & 59.6 & 40.4 & 37.1 & 8.4 \\
\hline
\end{tabular}

${ }^{\mathrm{a}}$ 7, tetramethylammonium bromide.

The mobile phase used for separation of products is 75/25- acetonitrile/water.

${ }^{b} \mathrm{HPLC}$ calibration curve for 1-ArOH: Area in $\mu \mathrm{V}=8.56 \times 10^{9}$ [1-ArOH] M - 34985 (5 points correlation coefficient $=0.99$ ); \% yield of 1$\mathrm{ArOH}=100 \times[1-\mathrm{ArOH}] \mathrm{M} /\left[1-\mathrm{ArN}_{2}{ }^{+}\right] \mathrm{M}$.

HPLC calibration curve for $1-\mathrm{ArBr}$ : Area in $\mu \mathrm{V}=1.36 \times 10^{10}$ [1-ArBr] M - 65016 (5 points correlation coefficient $\left.=0.99\right) ; \%$ yield of $1-\mathrm{ArBr}$ $=100 \times[1-\mathrm{ArBr}] \mathrm{M} /\left[1-\mathrm{ArN}_{2}{ }^{+}\right] \mathrm{M}$. Observed yields are reproducible within $\pm 1 \%$ in duplicate experiments.

${ }^{\mathrm{c}}$ Because of quantitative conversions with no significant extraneous peak, observed yields were normalized.

The obtained correlation between $\left[\mathrm{H}_{2} \mathrm{O}\right]$ and \%1-ArOH; $\left[\mathrm{Br}^{-}\right]$and $\% 1-\mathrm{ArBr}$ are following:
$\% 1-\mathrm{ArOH}=0.845\left[\mathrm{H}_{2} \mathrm{O}\right]^{1.17}$
(correlation coefficient $=0.99), \% 1-\mathrm{ArBr}=19.2\left[\mathrm{Br}^{-}\right]^{0.685}$
$($ correlation coefficient $=1.00)$ 


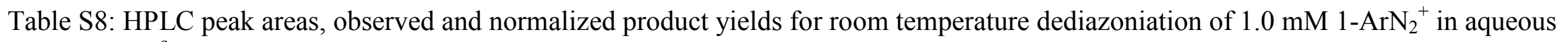
solutions of $\mathbf{8}^{\mathrm{a}}$ at $\mathrm{pH}=6.0$.

\begin{tabular}{|c|c|c|c|c|c|c|c|c|c|}
\hline \multirow{2}{*}{$\begin{array}{l}{[8]} \\
(\mathrm{M})\end{array}$} & \multicolumn{2}{|c|}{ Peak areas } & \multicolumn{3}{|c|}{ Observed yields ${ }^{\mathrm{b}}$} & \multicolumn{2}{|c|}{ Normalized yields ${ }^{\mathrm{c}}$} & \multirow{2}{*}{$\begin{array}{c}{\left[\mathrm{H}_{2} \mathrm{O}\right]} \\
(\mathrm{M})\end{array}$} & \multirow{2}{*}{$\mathrm{S}_{\mathrm{W}}{ }^{\mathrm{Br}}$} \\
\hline & $\begin{array}{c}1-\mathrm{ArOH} \\
\mu \mathrm{V}\end{array}$ & $\begin{array}{c}1-\mathrm{ArBr} \\
\mu \mathrm{V}\end{array}$ & $\begin{array}{c}1-\mathrm{ArOH} \\
(\%)\end{array}$ & $\begin{array}{c}1-\mathrm{ArBr} \\
(\%)\end{array}$ & $\begin{array}{l}\text { total } \\
(\%)\end{array}$ & $\begin{array}{c}1-\mathrm{ArOH} \\
(\%)\end{array}$ & $\begin{array}{c}1-\mathrm{ArBr} \\
(\%)\end{array}$ & & \\
\hline 0.1 & 2436059 & 124702 & 91.2 & 6.4 & 97.6 & 93.4 & 6.6 & 55.5 & 39.9 \\
\hline 0.5 & 2120367 & 404615 & 79.1 & 20.1 & 99.2 & 79.7 & 20.3 & 53.5 & 27.2 \\
\hline 1.0 & 1812013 & 615259 & 67.8 & 31.1 & 99.0 & 68.5 & 31.5 & 48.7 & 22.0 \\
\hline 1.5 & 1644013 & 783259 & 61.4 & 39.6 & 101.0 & 60.8 & 39.2 & 43.4 & 18.6 \\
\hline 2.0 & 1276013 & 1017259 & 47.4 & 50.6 & 98.0 & 48.4 & 51.6 & 40.1 & 21.4 \\
\hline 2.5 & 1100013 & 1215259 & 40.8 & 61.6 & 102.4 & 39.8 & 60.2 & 36.7 & 22.2 \\
\hline 3.0 & 916013 & 1293259 & 33.9 & 65.6 & 99.5 & 34.0 & 66.0 & 32.1 & 20.8 \\
\hline
\end{tabular}

${ }^{\mathrm{a}} \mathbf{8}, N$-Ethyl- $N, N$-dimethyl- $N$-propyl ammonium bromide.

The mobile phase used for separation of products is 70/30- acetonitrile/water.

${ }^{\mathrm{b}} \mathrm{HPLC}$ calibration curve for 1 - $\mathrm{ArOH}$ : Area in $\mu \mathrm{V}=8.0 \times 10^{9}$ [1-ArOH] $\mathrm{M}-28013$ (5 points correlation coefficient $=0.99$ ); $\%$ yield of 1 $\mathrm{ArOH}=100 \times[1-\mathrm{ArOH}] \mathrm{M} /\left[1-\mathrm{ArN}_{2}^{+}\right] \mathrm{M}$.

HPLC calibration curve for $1-\mathrm{ArBr}$ : Area in $\mu \mathrm{V}=6.0 \times 10^{9}$ [1-ArBr] M - 2741 (5 points correlation coefficient $\left.=0.99\right)$; $\%$ yield of 1 $\mathrm{ArBr}=100 \times[1-\mathrm{ArBr}] \mathrm{M} /\left[1-\mathrm{ArN}_{2}^{+}\right] \mathrm{M}$. Observed yields are reproducible within $\pm 1 \%$ in duplicate experiments.

${ }^{c}$ Because of quantitative conversions with no significant extraneous peak, observed yields were normalized.

The obtained correlation between $\left[\mathrm{H}_{2} \mathrm{O}\right]$ and $\% 1-\mathrm{ArOH} ;\left[\mathrm{Br}^{-}\right]$and $\% 1-\mathrm{ArBr}$ are following:

$$
\begin{aligned}
& \% 1-\mathrm{ArOH}=0.059\left[\mathrm{H}_{2} \mathrm{O}\right]^{1.822} \quad(\text { correlation coefficient }=0.99) \\
& \% 1-\mathrm{ArBr}=31.52\left[\mathrm{Br}^{-}\right]^{0.677} \quad(\text { correlation coefficient }=0.99)
\end{aligned}
$$




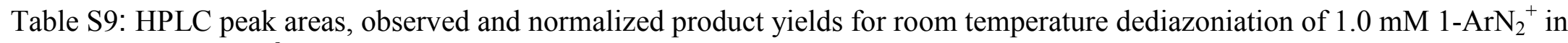
aqueous solutions of $\mathbf{9}^{\mathrm{a}}$ at $\mathrm{pH}=6.0$.

\begin{tabular}{|c|c|c|c|c|c|c|c|c|c|}
\hline \multirow{2}{*}{$\begin{array}{l}{[9]} \\
(\mathrm{M})\end{array}$} & \multicolumn{2}{|c|}{ Peak areas } & \multicolumn{3}{|c|}{ Observed yields ${ }^{\mathrm{b}}$} & \multicolumn{2}{|c|}{ Normalized yields ${ }^{\mathrm{c}}$} & \multirow{2}{*}{$\begin{array}{c}{\left[\mathrm{H}_{2} \mathrm{O}\right]} \\
(\mathrm{M})\end{array}$} & \multirow{2}{*}{$\mathrm{S}_{\mathrm{W}}{ }^{\mathrm{Br}}$} \\
\hline & $\begin{array}{c}1-\mathrm{ArOH} \\
\mu \mathrm{V}\end{array}$ & $\begin{array}{c}1-\mathrm{ArBr} \\
\mu \mathrm{V}\end{array}$ & $\begin{array}{c}\text { 1-ArOH } \\
(\%)\end{array}$ & $\begin{array}{c}1-\mathrm{ArBr} \\
(\%)\end{array}$ & $\begin{array}{r}\text { total } \\
(\%)\end{array}$ & $\begin{array}{c}1-\mathrm{ArOH} \\
(\%)\end{array}$ & $\begin{array}{c}1-\mathrm{ArBr} \\
(\%)\end{array}$ & & \\
\hline 0.1 & 2380013 & 94279 & 89.1 & 4.9 & 94.0 & 94.8 & 5.2 & 55.3 & 30.3 \\
\hline 0.5 & 2028013 & 323959 & 75.9 & 16.5 & 92.4 & 82.1 & 17.9 & 51.8 & 22.6 \\
\hline 1.0 & 1682110 & 676240 & 63.6 & 34.3 & 97.9 & 65.0 & 35.0 & 45.7 & 24.6 \\
\hline 1.5 & 1561843 & 790822 & 58.2 & 40.1 & 98.3 & 59.2 & 40.8 & 40.3 & 18.5 \\
\hline 2.0 & 1260013 & 1048639 & 46.9 & 53.1 & 100.0 & 46.9 & 53.1 & 35.9 & 20.3 \\
\hline 2.5 & 980013 & 1149259 & 36.1 & 58.4 & 94.5 & 38.2 & 61.8 & 31.5 & 20.4 \\
\hline 3.0 & 804173 & 1371259 & 29.4 & 69.6 & 99.0 & 29.7 & 70.3 & 26.5 & 20.9 \\
\hline
\end{tabular}

a, $N$-Ethyl- $N$-methyl- $N, N$-dipropylammoniumbromide.

The mobile phase used for separation of products is 70/30- acetonitrile/water.

${ }^{\mathrm{b}} \mathrm{HPLC}$ calibration curve for 1 - $\mathrm{ArOH}$ : Area in $\mu \mathrm{V}=8.0 \times 10^{9}$ [1-ArOH] M - 28013 (5 points correlation coefficient $=0.99$ ); $\%$ yield of 1 $\mathrm{ArOH}=100 \times[1-\mathrm{ArOH}] \mathrm{M} /\left[1-\mathrm{ArN}_{2}^{+}\right] \mathrm{M}$.

HPLC calibration curve for 1-ArBr: Area in $\mu \mathrm{V}=6.0 \times 10^{9}[1-\mathrm{ArBr}] \mathrm{M}-2741$ (5 points correlation coefficient $\left.=0.99\right)$; $\%$ yield of 1 $\mathrm{ArBr}=100 \times[1-\mathrm{ArBr}] \mathrm{M} /\left[1-\mathrm{ArN}_{2}{ }^{+}\right] \mathrm{M}$. Observed yields are reproducible within $\pm 1 \%$ in duplicate experiments.

${ }^{\mathrm{c}}$ Because of quantitative conversions with no significant extraneous peak, observed yields were normalized.

The obtained correlation between $\left[\mathrm{H}_{2} \mathrm{O}\right]$ and \%1-ArOH; $\left[\mathrm{Br}^{-}\right]$and \%1-ArBr are following:

$\% 1-\mathrm{ArOH}=0.186\left[\mathrm{H}_{2} \mathrm{O}\right]^{1 . .54}($ correlation coefficient $=0.99)$

$\% 1-\mathrm{ArBr}=31.1\left[\mathrm{Br}^{-}\right]^{0.77} \quad($ correlation coefficient $=0.99)$ 


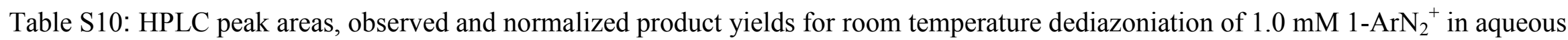
solutions of $\mathbf{1 0}^{\mathrm{a}}$ at $\mathrm{pH}=6.0$.

\begin{tabular}{|c|c|c|c|c|c|c|c|c|c|}
\hline \multirow{2}{*}{$\begin{array}{l}{[\mathbf{1 0}]} \\
(\mathrm{M})\end{array}$} & \multicolumn{2}{|c|}{ Peak areas } & \multicolumn{3}{|c|}{ Observed yields ${ }^{\mathrm{b}}$} & \multicolumn{2}{|c|}{ Normalized yields ${ }^{c}$} & \multirow{2}{*}{$\begin{array}{c}{\left[\mathrm{H}_{2} \mathrm{O}\right]} \\
(\mathrm{M})\end{array}$} & \multirow{2}{*}{$\mathrm{S}_{\mathrm{W}}{ }^{\mathrm{Br}}$} \\
\hline & $\begin{array}{c}1-\mathrm{ArOH} \\
\mu \mathrm{V}\end{array}$ & $\begin{array}{c}1-\mathrm{ArBr} \\
\mu \mathrm{V}\end{array}$ & $\begin{array}{c}1-\mathrm{ArOH} \\
(\%)\end{array}$ & $\begin{array}{c}1-\mathrm{ArBr} \\
(\%)\end{array}$ & $\begin{array}{r}\text { total } \\
(\%)\end{array}$ & $\begin{array}{c}1-\mathrm{ArOH} \\
(\%)\end{array}$ & $\begin{array}{c}1-\mathrm{ArBr} \\
(\%)\end{array}$ & & \\
\hline 0.1 & 1988797 & 100579 & 85.4 & 6.0 & 91.4 & 93.5 & 6.5 & 54.9 & 38.1 \\
\hline 0.5 & 1764013 & 329659 & 75.8 & 19.3 & 95.1 & 79.7 & 20.3 & 51.6 & 26.2 \\
\hline 1.0 & 1457731 & 554225 & 64.3 & 33.2 & 97.5 & 65.9 & 34.1 & 44.2 & 22.9 \\
\hline 1.5 & 1253208 & 636098 & 53.6 & 39.3 & 92.9 & 57.7 & 42.3 & 37.4 & 18.3 \\
\hline 2.0 & 1011615 & 848236 & 43.9 & 50.7 & 94.6 & 46.4 & 53.6 & 31.9 & 18.4 \\
\hline 2.5 & 889013 & 1044235 & 37.5 & 60.8 & 98.3 & 38.2 & 61.8 & 26.7 & 17.3 \\
\hline 3.0 & 631861 & 1102783 & 26.3 & 64.2 & 90.5 & 29.1 & 70.9 & 22.5 & 18.3 \\
\hline
\end{tabular}

a $\mathbf{1 0}, N, N, N$-tripropylammonium bromide.

The mobile phase used for separation of products is 70/30- acetonitrile/water.

${ }^{\mathrm{b}} \mathrm{HPLC}$ calibration curve for 1 - $\mathrm{ArOH}$ : Area in $\mu \mathrm{V}=8.0 \times 10^{9}$ [1-ArOH] M - 28013 (5 points correlation coefficient $=0.99$ ); \% yield of 1 $\mathrm{ArOH}=100 \times[1-\mathrm{ArOH}] \mathrm{M} /\left[1-\mathrm{ArN}_{2}^{+}\right] \mathrm{M}$.

HPLC calibration curve for 1-ArBr: Area in $\mu \mathrm{V}=6.0 \times 10^{9}[1-\mathrm{ArBr}] \mathrm{M}-2741$ (5 points correlation coefficient $\left.=0.99\right)$; \% yield of $1-$ $\mathrm{ArBr}=100 \times[1-\mathrm{ArBr}] \mathrm{M} /\left[1-\mathrm{ArN}_{2}{ }^{+}\right] \mathrm{M}$. Observed yields are reproducible within $\pm 1 \%$ in duplicate experiments.

${ }^{\mathrm{c}}$ Because of quantitative conversions with no significant extraneous peak, observed yields were normalized.

The obtained correlation between $\left[\mathrm{H}_{2} \mathrm{O}\right]$ and \%1-ArOH; $\left[\mathrm{Br}^{-}\right]$and $\% 1-\mathrm{ArBr}$ are following:

$\% 1-\mathrm{ArOH}=0.66\left[\mathrm{H}_{2} \mathrm{O}\right]^{1.226} \quad($ correlation coefficient $=0.99)$

$\% 1-\mathrm{ArBr}=32.8\left[\mathrm{Br}^{-}\right]^{0.70} \quad($ correlation coefficient $=0.99)$ 
Table S11: HPLC peak areas, observed and normalized product yields for room temperature dediazoniation of $1.0 \mathrm{mM} 1$-ArN $_{2}^{+}$in aqueous solutions of $11^{\mathrm{a}}$ at $\mathrm{pH}=6.0$.

\begin{tabular}{|c|c|c|c|c|c|c|c|c|c|}
\hline \multirow{2}{*}{$\begin{array}{l}{[11]} \\
(\mathrm{M})\end{array}$} & \multicolumn{2}{|c|}{ Peak areas } & \multicolumn{3}{|c|}{ Observed yields ${ }^{b}$} & \multicolumn{2}{|c|}{ Normalized yields ${ }^{\mathrm{c}}$} & \multirow{2}{*}{$\begin{array}{c}{\left[\mathrm{H}_{2} \mathrm{O}\right]} \\
(\mathrm{M})\end{array}$} & \multirow{2}{*}{$\mathrm{S}_{\mathrm{W}}{ }^{\mathrm{Br}}$} \\
\hline & $\begin{array}{c}\text { 1-ArOH } \\
\mu \mathrm{V}\end{array}$ & $\begin{array}{c}1-\mathrm{ArBr} \\
\mu \mathrm{V}\end{array}$ & $\begin{array}{c}\text { 1-ArOH } \\
(\%)\end{array}$ & $\begin{array}{c}1-\mathrm{ArBr} \\
(\%)\end{array}$ & $\begin{array}{r}\text { total } \\
(\%)\end{array}$ & $\begin{array}{c}\text { 1-ArOH } \\
(\%)\end{array}$ & $\begin{array}{c}1-\mathrm{ArBr} \\
(\%)\end{array}$ & & \\
\hline 0.1 & 2302615 & 93629 & 90.0 & 3.2 & 93.2 & 96.6 & 3.4 & 54.5 & 19.2 \\
\hline 0.5 & 2122060 & 383316 & 83.3 & 10.0 & 93.3 & 89.3 & 10.7 & 51.0 & 12.2 \\
\hline 1.0 & 2161371 & 778559 & 83.3 & 19.7 & 103.0 & 80.9 & 19.1 & 47.1 & 11.1 \\
\hline 1.5 & 2121455 & 1086888 & 82.7 & 21.3 & 104.0 & 75.2 & 24.8 & 43.5 & 9.6 \\
\hline 2.0 & 1747302 & 1157833 & 66.9 & 29.1 & 96.0 & 69.8 & 30.2 & 39.7 & 8.6 \\
\hline 2.5 & 1493818 & 1406713 & 58.8 & 35.2 & 94.0 & 62.6 & 37.4 & 36.2 & 8.6 \\
\hline 3.0 & 1338658 & 1901753 & 52.7 & 47.3 & 93.0 & 56.7 & 43.3 & 31.2 & 7.9 \\
\hline
\end{tabular}

${ }^{a} \mathbf{1 1}, N, N$-Dimethyl- $N$-ethyl- $N$-(2-hydroxyethyl)ammonium bromide.

The mobile phase used for separation of products is 75/25- acetonitrile/water.

${ }^{\mathrm{b}} \mathrm{HPLC}$ calibration curve for 1 - ArOH: Area in $\mu \mathrm{V}=8.62 \times 10^{9}$ [1-ArOH] M -34985 (5 points correlation coefficient $=0.999$ ); $\%$ yield of 1 $\mathrm{ArOH}=100 \times[1-\mathrm{ArOH}] \mathrm{M} /\left[1-\mathrm{ArN}_{2}^{+}\right] \mathrm{M}$.

HPLC calibration curve for 1 -ArBr: Area in $\mu \mathrm{V}=1.36 \times 10^{10}[1-\mathrm{ArBr}] \mathrm{M}-65016$ (5 points correlation coefficient $\left.=0.999\right)$; $\%$ yield of $1-$ $\mathrm{ArBr}=100 \times[1-\mathrm{ArBr}] \mathrm{M} /\left[1-\mathrm{ArN}_{2}^{+}\right] \mathrm{M}$. Observed yields are reproducible within $\pm 1 \%$ in duplicate experiments.

${ }^{\mathrm{c}}$ Because of quantitative conversions with no significant extraneous peak, observed yields were normalized.

The obtained correlation between $\left[\mathrm{H}_{2} \mathrm{O}\right]$ and \%1-ArOH; $\left[\mathrm{Br}^{-}\right]$and $\% 1-\mathrm{ArBr}$ are following:

$\% 1-\mathrm{ArOH}=2.0\left[\mathrm{H}_{2} \mathrm{O}\right]^{0.96} \quad($ correlation coefficient $=0.99)$

$\% 1-\mathrm{ArBr}=18.6\left[\mathrm{Br}^{-}\right]^{0.75} \quad($ correlation coefficient $=0.99)$ 


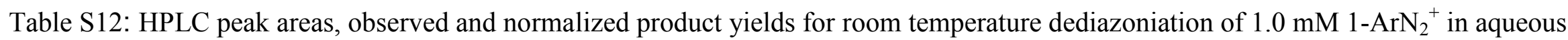
solutions of $12^{\mathrm{a}}$ at $\mathrm{pH}=6.0$.

\begin{tabular}{|c|c|c|c|c|c|c|c|c|c|}
\hline \multirow{2}{*}{$\begin{array}{l}{[12]} \\
(\mathrm{M})\end{array}$} & \multicolumn{2}{|c|}{ Peak areas } & \multicolumn{3}{|c|}{ Observed yields ${ }^{b}$} & \multicolumn{2}{|c|}{ Normalized yields ${ }^{c}$} & \multirow{2}{*}{$\begin{array}{c}{\left[\mathrm{H}_{2} \mathrm{O}\right]} \\
(\mathrm{M})\end{array}$} & \multirow{2}{*}{$\mathrm{S}_{\mathrm{W}}{ }^{\mathrm{Br}}$} \\
\hline & $\begin{array}{c}1-\mathrm{ArOH} \\
\mu \mathrm{V}\end{array}$ & $\begin{array}{c}1-\mathrm{ArBr} \\
\mu \mathrm{V}\end{array}$ & $\begin{array}{c}\text { 1-ArOH } \\
(\%)\end{array}$ & $\begin{array}{c}1-\mathrm{ArBr} \\
(\%)\end{array}$ & $\begin{array}{r}\text { total } \\
(\%)\end{array}$ & $\begin{array}{c}\text { 1-ArOH } \\
(\%)\end{array}$ & $\begin{array}{c}\text { 1-ArBr } \\
(\%)\end{array}$ & & \\
\hline 0.1 & 2591322 & 143468 & 88.8 & 7.1 & 95.9 & 92.6 & 7.4 & 54.8 & 43.8 \\
\hline 0.5 & 2158142 & 428072 & 73.7 & 21.9 & 95.6 & 77.1 & 22.9 & 52.4 & 31.1 \\
\hline 1.0 & 1745322 & 679268 & 59.5 & 35.1 & 94.6 & 63.0 & 37.0 & 47.2 & 27.7 \\
\hline 1.5 & 1642863 & 925936 & 56.2 & 47.8 & 104.0 & 54.0 & 46.0 & 42.8 & 24.3 \\
\hline 2.0 & 1259322 & 955268 & 42.6 & 49.5 & 92.1 & 46.3 & 53.7 & 38.6 & 22.4 \\
\hline 2.5 & 1079322 & 1093268 & 36.4 & 56.7 & 93.1 & 39.1 & 60.9 & 34.6 & 21.5 \\
\hline 3.0 & 953322 & 1285268 & 31.9 & 66.5 & 98.4 & 32.4 & 67.6 & 30.2 & 21.0 \\
\hline
\end{tabular}

${ }^{a}$ 12, $N$-(2-methoxyethyl)- $N, N$-dimethylammonium bromide.

The mobile phase used for separation of products is 70/30- acetonitrile/water.

${ }^{\mathrm{b}} \mathrm{HPLC}$ calibration curve for 1 - ArOH: Area in $\mu \mathrm{V}=9.0 \times 10^{9}$ [1-ArOH] M 35322 (5 points correlation coefficient $\left.=0.99\right) ; \%$ yield of 1 $\mathrm{ArOH}=100 \times[1-\mathrm{ArOH}] \mathrm{M} /\left[1-\mathrm{ArN}_{2}^{+}\right] \mathrm{M}$.

HPLC calibration curve for $1-\mathrm{ArBr}$ : Area in $\mu \mathrm{V}=6.0 \times 10^{9}[1-\mathrm{ArBr}] \mathrm{M}+7268(5$ points correlation coefficient $=0.99)$; $\%$ yield of $1-$ $\mathrm{ArBr}=100 \times[1-\mathrm{ArBr}] \mathrm{M} /\left[1-\mathrm{ArN}_{2}{ }^{+}\right] \mathrm{M}$. Observed yields are reproducible within $\pm 1 \%$ in duplicate experiments.

${ }^{\mathrm{c}}$ Because of quantitative conversions with no significant extraneous peak, observed yields were normalized.

The obtained correlation between $\left[\mathrm{H}_{2} \mathrm{O}\right]$ and \%1-ArOH; $\left[\mathrm{Br}^{-}\right]$and $\% 1-\mathrm{ArBr}$ are following:

$\% 1-\mathrm{ArOH}=0.099\left[\mathrm{H}_{2} \mathrm{O}\right]^{1.69}($ correlation coefficient $=0.98)$

$\% 1-\mathrm{ArBr}=34.57\left[\mathrm{Br}^{-}\right]^{0.65} \quad($ correlation coefficient $=0.99)$ 
Table S13: HPLC peak areas, observed and normalized product yields for room temperature dediazoniation of $1.0 \mathrm{mM} 16-\mathrm{ArN}_{2}{ }^{+}$in $\mathrm{w} / \mathrm{o}$ microemulsions of $\mathbf{1}^{\mathrm{a}}$ at $\mathrm{pH}=6.0$.

\begin{tabular}{|c|c|c|c|c|c|c|c|c|c|c|c|c|c|}
\hline \multirow{2}{*}{$\mathrm{W}_{0}$} & \multicolumn{3}{|c|}{ Peak areas } & \multicolumn{4}{|c|}{ Observed yields ${ }^{\mathrm{b}}$} & \multicolumn{3}{|c|}{ Normalized yields ${ }^{c}$} & \multirow{2}{*}{$\begin{array}{c}{\left[\mathrm{H}_{2} \mathrm{O}_{\mathrm{i}}\right]} \\
(\mathrm{M})\end{array}$} & \multirow{2}{*}{$\begin{array}{c}{\left[\mathrm{Br}_{\mathrm{i}}^{-}\right]} \\
(\mathrm{M})\end{array}$} & \multirow{2}{*}{$\begin{array}{c}{\left[\mathrm{HexOH}_{\mathrm{I}}\right]} \\
(\mathrm{M})\end{array}$} \\
\hline & $\begin{array}{c}\text { 16-ArOH } \\
\mu \mathrm{V}\end{array}$ & $\begin{array}{c}16-\mathrm{ArBr} \\
\mu \mathrm{V}\end{array}$ & $\begin{array}{c}\text { 16-ArOHex } \\
\mu \mathrm{V}\end{array}$ & $\begin{array}{c}\text { 16-ArOH } \\
(\%)\end{array}$ & $\begin{array}{c}16-\mathrm{ArBr} \\
(\%)\end{array}$ & $\begin{array}{c}\text { 16-ArOHex } \\
(\%)\end{array}$ & $\begin{array}{r}\text { total } \\
(\%)\end{array}$ & $\begin{array}{l}\text { 16-ArOH } \\
(\%)\end{array}$ & $\begin{array}{c}16-\mathrm{ArBr} \\
(\%)\end{array}$ & $\begin{array}{c}\text { 16-ArOHex } \\
(\%)\end{array}$ & & & \\
\hline 40 & 2007358 & 1651852 & 118064 & 54.0 & 44.4 & 3.6 & 102.0 & 52.7 & 43.3 & 4.0 & 32.6 & 3.28 & 3.4 \\
\hline 48 & 1684122 & 1381840 & 118523 & 52.8 & 33.3 & 4.2 & 90.3 & 58.4 & 36.8 & 4.8 & 33.4 & 2.58 & 4.0 \\
\hline 56 & 2415432 & 1972205 & 176360 & 58.6 & 36.5 & 4.7 & 99.8 & 58.7 & 36.6 & 4.7 & 33.5 & 2.56 & 3.9 \\
\hline
\end{tabular}

${ }^{\mathrm{a}} \mathbf{1}$, Cetyltrimethyl ammonium bromide.

The mobile phase used for separation of products is 72/28- methanol/isopropanol.

${ }^{\mathrm{b}} \mathrm{HPLC}$ calibration curve for $16-\mathrm{ArOH}$ : Area in $\mu \mathrm{V}=3.0 \times 10^{10}$ [16-ArOH] M - 28188 (5 points correlation coefficient $=0.99$ ); $\%$ yield of $16-\mathrm{ArOH}=100 \times[16-\mathrm{ArOH}] \mathrm{M} /\left[16-\mathrm{ArN}_{2}^{+}\right] \mathrm{M}$.

HPLC calibration curve for $16-\mathrm{ArBr}$ : Area in $\mu \mathrm{V}=4.0 \times 10^{10}[16-\mathrm{ArBr}] \mathrm{M}-57195$ (5 points correlation coefficient $\left.=0.99\right)$; $\%$ yield of $16-$ $\mathrm{ArBr}=100 \times[16-\mathrm{ArBr}] \mathrm{M} /\left[16-\mathrm{ArN}_{2}{ }^{+}\right] \mathrm{M}$.

HPLC calibration curve for 16-ArOHex: Area in $\mu \mathrm{V}=3.0 \times 10^{10}[16-\mathrm{ArOHex}] \mathrm{M}-19630$ (5 points correlation coefficient $\left.=0.99\right)$; $\%$ yield of $16-\mathrm{ArOHex}=100 \times[16-\mathrm{ArOHex}] \mathrm{M} /\left[16-\mathrm{ArN}_{2}{ }^{+}\right] \mathrm{M}$. Observed yields are reproducible within $\pm 1 \%$ in duplicate experiments.

${ }^{\mathrm{c}}$ Because of quantitative conversions with no significant extraneous peak, observed yields were normalized and normalized yields were used to estimate the interfacial concentrations.

The correlation equation used between $\left[\mathrm{Br}_{\mathrm{i}}^{-}\right]$and $\% 16-\mathrm{ArBr} ;\left[\mathrm{HexOH}_{\mathrm{i}}\right]$ and $\% 16-\mathrm{ArOHex}$ are following:

$\% 16-\mathrm{ArBr}=19.2\left[\mathrm{Br}_{\mathrm{i}}^{-}\right]^{0.685} \quad$ (correlation coefficient $\left.=1.00\right), \% 16-\mathrm{ArOHex}=1.214\left[\mathrm{HexOH}_{\mathrm{i}}\right]-0.1 \quad$ (correlation coefficient $\left.=0.99\right)$. 


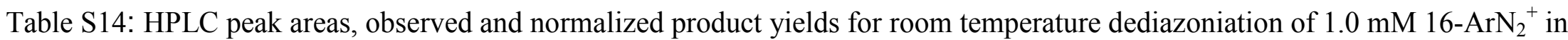
w/o microemulsions of $2^{\mathrm{a}}$ at $\mathrm{pH}=6.0$.

\begin{tabular}{|c|c|c|c|c|c|c|c|c|c|c|c|c|c|}
\hline \multirow{2}{*}{$\mathrm{W}_{0}$} & \multicolumn{3}{|c|}{ Peak areas } & \multicolumn{4}{|c|}{ Observed yields ${ }^{b}$} & \multicolumn{3}{|c|}{ Normalized yields ${ }^{c}$} & \multirow{2}{*}{$\begin{array}{l}{\left[\mathrm{H}_{2} \mathrm{O}_{\mathrm{i}}\right]} \\
(\mathrm{M})\end{array}$} & \multirow{2}{*}{$\begin{array}{c}{\left[\mathrm{Br}_{\mathrm{i}}^{-}\right]} \\
(\mathrm{M})\end{array}$} & \multirow{2}{*}{$\begin{array}{c}{\left[\mathrm{HexOH}_{\mathrm{i}}\right]} \\
(\mathrm{M})\end{array}$} \\
\hline & $\begin{array}{c}\text { 16-ArOH } \\
\mu \mathrm{V}\end{array}$ & $\begin{array}{c}\text { 16-ArBr } \\
\mu \mathrm{V}\end{array}$ & $\begin{array}{c}\text { 16-ArOHex } \\
\mu \mathrm{V}\end{array}$ & $\begin{array}{c}\text { 16-ArOH } \\
(\%)\end{array}$ & $\begin{array}{c}16-\mathrm{ArBr} \\
(\%)\end{array}$ & $\begin{array}{c}\text { 16-ArOHex } \\
(\%)\end{array}$ & $\begin{array}{l}\text { total } \\
(\%)\end{array}$ & $\begin{array}{c}\text { 16-ArOH } \\
(\%)\end{array}$ & $\begin{array}{c}16-\mathrm{ArBr} \\
(\%)\end{array}$ & $\begin{array}{c}\text { 16-ArOHex } \\
(\%)\end{array}$ & & & \\
\hline 8 & 1589652 & 2360475 & 176930 & 42.8 & 45.7 & 5.2 & 93.7 & 45.7 & 48.7 & 5.6 & 31.8 & 1.9 & 4.7 \\
\hline 16 & 1506089 & 1518890 & 106834 & 51.1 & 39.4 & 4.2 & 94.7 & 53.9 & 41.6 & 4.5 & 35.0 & 1.5 & 3.8 \\
\hline 24 & 2310483 & 1964966 & 168543 & 57.7 & 37.4 & 4.6 & 99.7 & 57.9 & 37.5 & 4.6 & 35.8 & 1.3 & 3.9 \\
\hline 32 & 2243592 & 1747125 & 150470 & 60.1 & 35.8 & 4.5 & 100.4 & 59.9 & 35.7 & 4.4 & 36.3 & 1.2 & 3.7 \\
\hline 40 & 2327412 & 1700405 & 128570 & 60.4 & 33.8 & 3.8 & 98.0 & 61.6 & 34.5 & 3.9 & 35.6 & 1.1 & 3.3 \\
\hline 48 & 2112281 & 1509757 & 153228 & 63.7 & 35.0 & 5.1 & 103.8 & 61.3 & 33.7 & 5.0 & 36.2 & 1.1 & 4.2 \\
\hline 56 & 1843335 & 1247569 & 109814 & 55.4 & 29.1 & 3.8 & 88.4 & 62.7 & 32.9 & 4.4 & 34.6 & 1.0 & 3.7 \\
\hline $\begin{array}{l}{ }^{\mathrm{a}} \boldsymbol{2}, \\
\mathrm{Th} \\
{ }_{\mathrm{b}} \mathrm{H} \\
16- \\
\mathrm{HP} \\
\mathrm{Ar} \\
\mathrm{Hl} \\
16- \\
{ }^{\mathrm{c}} \mathrm{B} \\
\text { to }\end{array}$ & $\begin{array}{l}N \text {-Hexadecy } \\
\text { e mobile pha } \\
\text { PLC calibra } \\
\text { ArOH }=100 \times \\
\text { LC calibrati } \\
3 r=100 \times[16 \\
\text { LC calibrat } \\
\text { ArOHex }=1 \\
\text { ecause of qu } \\
\text { estimate the }\end{array}$ & $\begin{array}{l}\text { yl- } N, N \text {-dim } \\
\text { ase used for } \\
\text { ation curve } \\
\times[16-\mathrm{ArOH} \\
\text { ion curve } \mathrm{ft} \\
6-\mathrm{ArBr}] \mathrm{M} /[ \\
\text { ion curve } \mathrm{f} \\
00 \times[16-\mathrm{ArC} \\
\text { dantitative } \\
\text { interfacial }\end{array}$ & $\begin{array}{l}\text { ethyl- } N \text {-propyl } \\
\text { separation of } \\
\text { for } 16-\mathrm{ArOH} \\
\text { ] } \mathrm{M} /\left[16-\mathrm{ArN}{ }_{2}\right. \\
\text { or } 16-\mathrm{ArBr}: \mathrm{A} \\
\left.16-\mathrm{ArN}_{2}^{+}\right] \mathrm{M} \text {. } \\
\text { or } 16-\mathrm{ArOHex} \\
\mathrm{OHex}] \mathrm{M} /[16- \\
\text { conversions wi } \\
\text { concentrations. }\end{array}$ & $\begin{array}{l}\text { ammonium } \\
\text { products is } \\
\text { : Area in } \mu \\
\text { ] } \mathrm{M} \text {. } \\
\text { ea in } \mu \mathrm{V}= \\
\text { Area in } \mu \mathrm{V} \\
\left.\mathrm{ArN}_{2}^{+}\right] \mathrm{M} \text {. O } \\
\text { th no signifi }\end{array}$ & $\begin{array}{l}\text { bromide. } \\
72 / 28-\text { met } \\
V=3.0 \times 10 \\
=4.0 \times 10^{10} \\
V=3.0 \times 10 \\
\text { Observed yi } \\
\text { icant extra }\end{array}$ & $\begin{array}{l}\text { hanol/isoprop } \\
{ }^{10}[16-\mathrm{ArOH}] \\
16-\mathrm{ArBr}] \mathrm{M} \\
{ }^{10} \text { [16-ArOHex } \\
\text { ields are repro } \\
\text { neous peak, ol }\end{array}$ & $\begin{array}{l}\text { anol. } \\
\text { M - } 281 \\
57195 \\
\text { x] M - 1 } \\
\text { ducible } \\
\text { oserved }\end{array}$ & $\begin{array}{l}88 \text { (5 points } \\
(5 \text { points cc } \\
630(5 \text { poil } \\
\text { vithin } \pm 1 \% \\
\text { jields were }\end{array}$ & $\begin{array}{l}\text { s correlati } \\
\text { rrelation } \\
\text { in duplic } \\
\text { normalize }\end{array}$ & $\begin{array}{l}\text { coefficient } \\
\text { tion coefficient }=0 \\
\text { te experiment } \\
\text { d and normali }\end{array}$ & $\begin{array}{l}0.99) ; \% \\
9) ; \% \text { yi } \\
=0.99) ; \\
\text { d yields }\end{array}$ & $\begin{array}{l}\text { yield } \\
\text { eld of } 1 \\
\text { o yield } \\
\text { were us }\end{array}$ & $\begin{array}{l}\text { of } \\
6- \\
\text { of } \\
\text { ed }\end{array}$ \\
\hline
\end{tabular}


Table S15: HPLC peak areas, observed and normalized product yields for room temperature dediazoniation of $1.0 \mathrm{mM} 16-\mathrm{ArN}_{2}{ }^{+}$in $\mathrm{w} / \mathrm{o}$ microemulsions of $\mathbf{3}^{\mathrm{a}}$ at $\mathrm{pH}=6.0$.

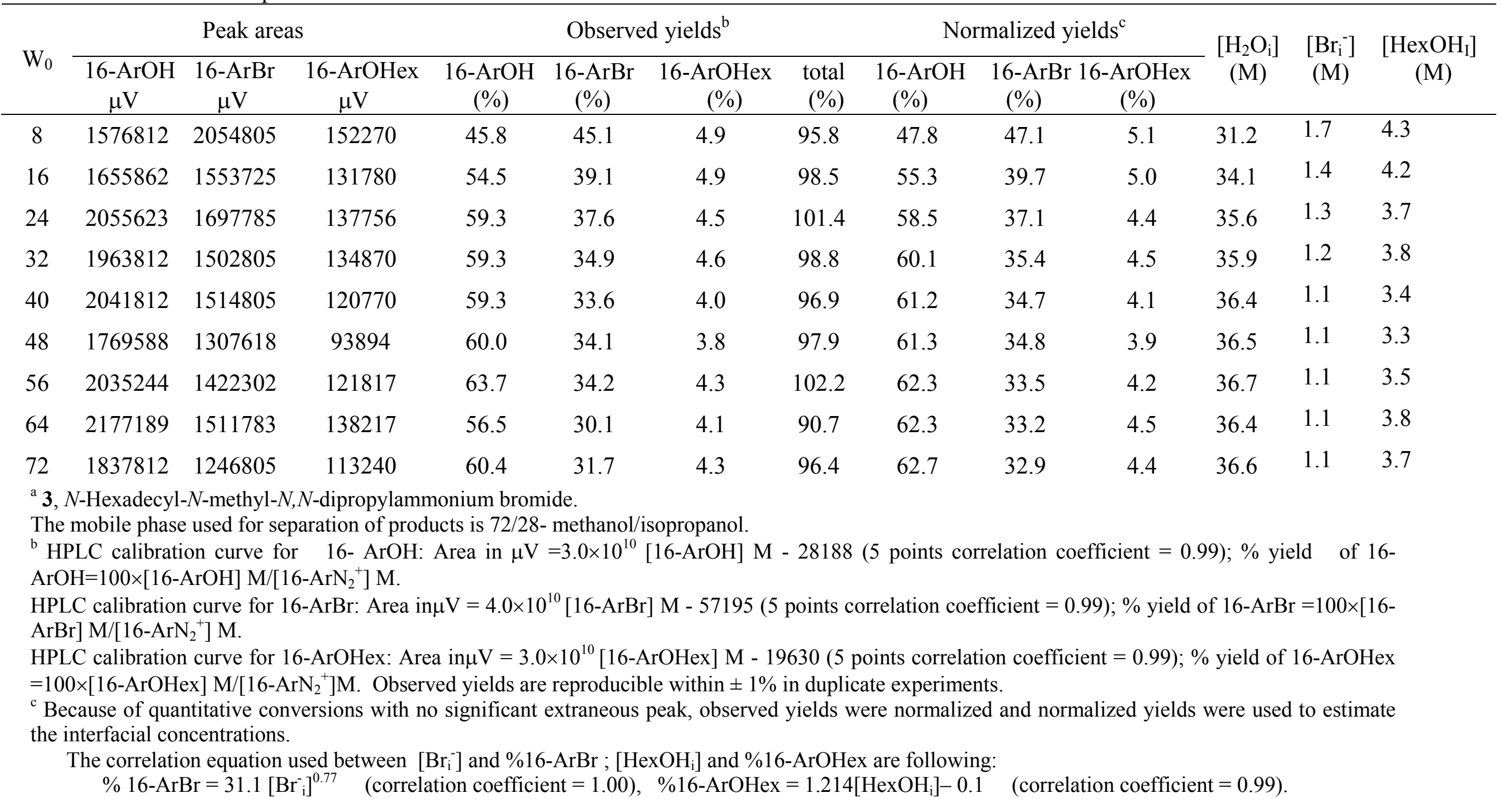


Table S16: HPLC peak areas, observed and normalized product yields for room temperature dediazoniation of $1.0 \mathrm{mM} 16-\mathrm{ArN}_{2}{ }^{+}$in $\mathrm{w} / \mathrm{o}$ microemulsions of $4^{\mathrm{a}}$ at $\mathrm{pH}=6.0$.

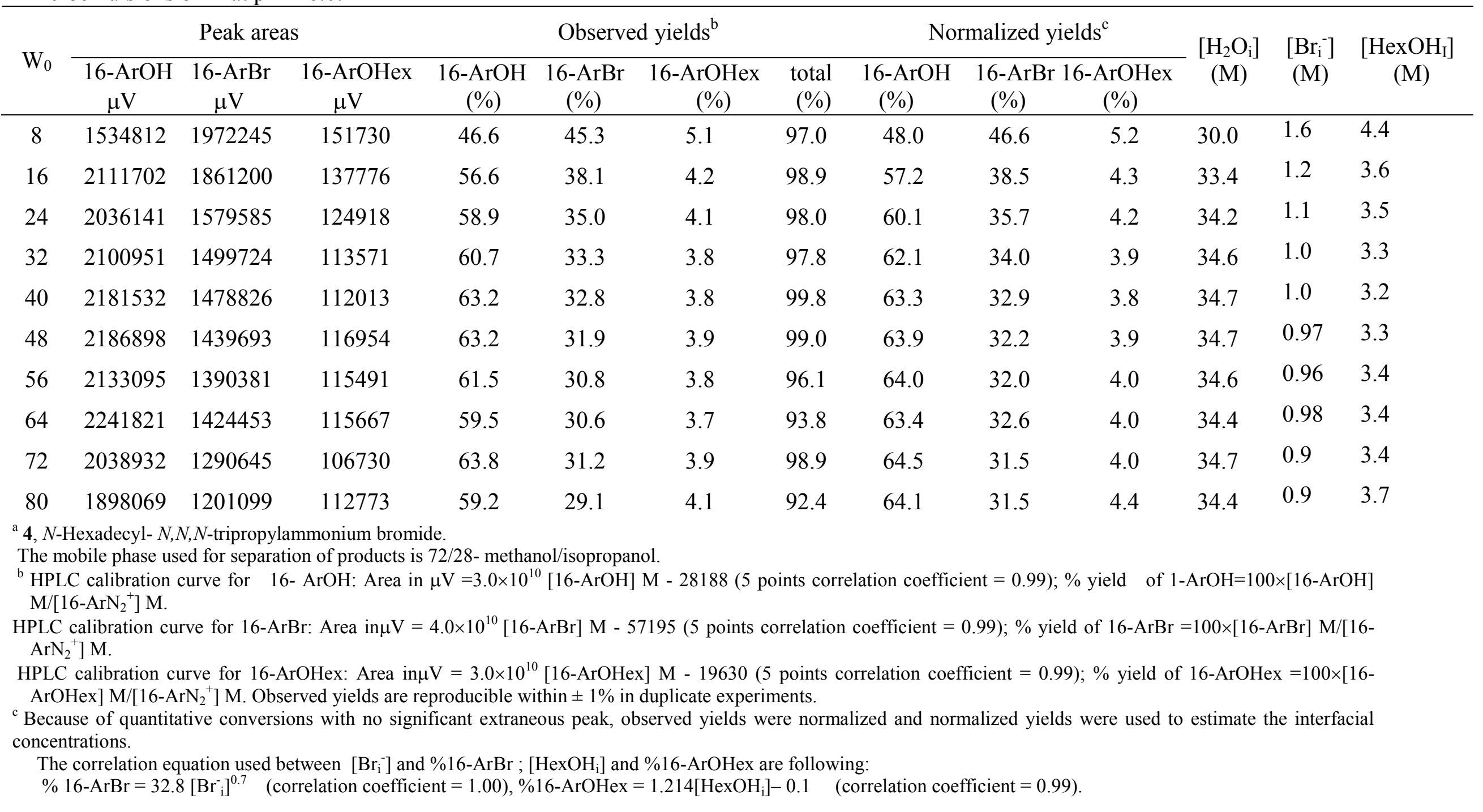


Table S17: HPLC peak areas, observed and normalized product yields for room temperature dediazoniation of $1.0 \mathrm{mM}^{16-\mathrm{ArN}_{2}}{ }^{+}$in $\mathrm{w} / \mathrm{o}$ microemulsions of $\mathbf{5}^{\mathrm{a}}$ at $\mathrm{pH}=6.0$.

\begin{tabular}{|c|c|c|c|c|c|c|c|c|c|c|c|c|c|}
\hline \multirow{2}{*}{$\mathrm{W}_{0}$} & \multicolumn{3}{|c|}{ Peak areas } & \multicolumn{4}{|c|}{ Observed yields ${ }^{\mathrm{b}}$} & \multicolumn{3}{|c|}{ Normalized yields $\mathrm{c}$} & \multirow{2}{*}{$\begin{array}{c}{\left[\mathrm{H}_{2} \mathrm{O}_{\mathrm{i}}\right]} \\
(\mathrm{M})\end{array}$} & \multirow{2}{*}{$\begin{array}{c}{\left[\mathrm{Br}_{\mathrm{i}}^{-}\right]} \\
(\mathrm{M})\end{array}$} & \multirow{2}{*}{$\begin{array}{c}{\left[\mathrm{HexOH}_{\mathrm{i}}\right.} \\
(\mathrm{M})\end{array}$} \\
\hline & $\begin{array}{c}\text { 16-ArOH } \\
\mu \mathrm{V}\end{array}$ & $\begin{array}{c}16-\mathrm{ArBr} \\
\mu \mathrm{V}\end{array}$ & $\begin{array}{c}\text { 16-ArOHex } \\
\mu \mathrm{V}\end{array}$ & $\begin{array}{c}\text { 16-ArOH } \\
(\%)\end{array}$ & $\begin{array}{c}16-\mathrm{ArBr} \\
(\%)\end{array}$ & $\begin{array}{c}\text { 16-ArOHex } \\
(\%)\end{array}$ & $\begin{array}{r}\text { total } \\
(\%)\end{array}$ & $\begin{array}{c}\text { 16-ArOH } \\
(\%)\end{array}$ & $\begin{array}{c}\text { 16-ArBr } \\
(\%)\end{array}$ & $\begin{array}{c}\text { 16-ArOHex } \\
(\%)\end{array}$ & & & \\
\hline 40 & 2065446 & 1748193 & 144794 & 56.3 & 36.4 & 4.4 & 97.1 & 57.9 & 37.5 & 4.6 & 33.1 & 2.5 & 3.9 \\
\hline 48 & 1711721 & 1459911 & 123952 & 58.6 & 38.4 & 4.8 & 101.8 & 57.6 & 37.7 & 4.7 & 33.0 & 2.6 & 3.9 \\
\hline 56 & 2236416 & 1806244 & 154822 & 60.9 & 37.6 & 4.7 & 103.2 & 59.0 & 36.4 & 4.6 & 33.4 & 2.4 & 3.9 \\
\hline
\end{tabular}

a 5 -Hexadecyl- $N$-(2-hydroxyethyl)- $N, N$-dimethylammonium bromide.

The mobile phase used for separation of products is 72/28- methanol/isopropanol.

${ }^{\mathrm{b}}$ HPLC calibration curve for $16-\mathrm{ArOH}$ : Area in $\mu \mathrm{V}=3.0 \times 10^{10}[16-\mathrm{ArOH}] \mathrm{M}-28188$ (5 points correlation coefficient $\left.=0.99\right)$; $\%$ yield of $16-$ $\mathrm{ArOH}=100 \times[16-\mathrm{ArOH}] \mathrm{M} /\left[16-\mathrm{ArN}_{2}^{+}\right] \mathrm{M}$.

HPLC calibration curve for 16-ArBr: Area in $\mu \mathrm{V}=4.0 \times 10^{10}[16-\mathrm{ArBr}] \mathrm{M}-57195$ (5 points correlation coefficient $\left.=0.99\right) ; \%$ yield of $16-\mathrm{ArBr}$ $=100 \times[16-\mathrm{ArBr}] \mathrm{M} /\left[16-\mathrm{ArN}_{2}^{+}\right] \mathrm{M}$.

HPLC calibration curve for 16-ArOHex: Area in $\mu \mathrm{V}=3.0 \times 10^{10}$ [16-ArOHex] M - 19630 (5 points correlation coefficient = 0.99); \% yield of 16ArOHex $=100 \times[16-\mathrm{ArOHex}] \mathrm{M} /\left[16-\mathrm{ArN}_{2}^{+}\right] \mathrm{M}$ Observed yields are reproducible within $\pm 1 \%$ in duplicate experiments.

${ }^{\mathrm{c}}$ Because of quantitative conversions with no significant extraneous peak, observed yields were normalized and normalized yields were used to estimate the interfacial concentrations.

The correlation equation used between $\left[\mathrm{Br}_{\mathrm{i}}^{-}\right]$and $\% 16-\mathrm{ArBr} ;\left[\mathrm{HexOH}_{\mathrm{i}}\right]$ and $\% 16-\mathrm{ArOHex}$ are following:

$\% 16-\mathrm{ArBr}=18.6\left[\mathrm{Br}_{\mathrm{i}}^{-}\right]^{0.75} \quad($ correlation coefficient $=1.00), \% 16-\mathrm{ArOHex}=1.214\left[\mathrm{HexOH}_{\mathrm{i}}\right]-0.1 \quad($ correlation coefficient $=0.99)$. 


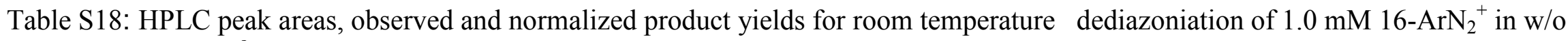
microemulsions of $6^{\mathrm{a}}$ at $\mathrm{pH}=6.0$.

\begin{tabular}{|c|c|c|c|c|c|c|c|c|c|c|c|c|c|}
\hline \multirow{2}{*}{$\mathrm{W}_{0}$} & \multicolumn{3}{|c|}{ Peak areas } & \multicolumn{4}{|c|}{ Observed yields ${ }^{b}$} & \multicolumn{3}{|c|}{ Normalized yields ${ }^{c}$} & \multirow{2}{*}{$\begin{array}{c}{\left[\mathrm{H}_{2} \mathrm{O}_{\mathrm{i}}\right]} \\
(\mathrm{M})\end{array}$} & \multirow{2}{*}{$\begin{array}{c}{\left[\mathrm{Br}_{\mathrm{i}}^{-}\right]} \\
(\mathrm{M})\end{array}$} & \multirow{2}{*}{$\begin{array}{c}{\left[\mathrm{HexOH}_{\mathrm{I}}\right]} \\
(\mathrm{M})\end{array}$} \\
\hline & $\begin{array}{c}\text { 16-ArOH } \\
\mu \mathrm{V}\end{array}$ & $\begin{array}{c}16-\mathrm{ArBr} \\
\mu \mathrm{V}\end{array}$ & $\begin{array}{c}\text { 16-ArOHex } \\
\mu \mathrm{V}\end{array}$ & $\begin{array}{c}\text { 16-ArOH } \\
(\%)\end{array}$ & $\begin{array}{c}16-\mathrm{ArBr} \\
(\%)\end{array}$ & $\begin{array}{c}\text { 16-ArOHex } \\
(\%)\end{array}$ & $\begin{array}{r}\text { total } \\
(\%)\end{array}$ & $\begin{array}{l}\text { 16-ArOH } \\
(\%)\end{array}$ & $\begin{array}{c}16-\mathrm{ArBr} \\
(\%)\end{array}$ & $\begin{array}{c}\text { 16-ArOHex } \\
(\%)\end{array}$ & & & \\
\hline 32 & 1631431 & 1128749 & 92553 & 58.1 & 31.1 & 3.9 & 93.1 & 62.4 & 33.4 & 4.2 & 41.5 & 0.95 & 3.54 \\
\hline 40 & 2673484 & 1782610 & 149339 & 66.2 & 33.8 & 4.1 & 104.1 & 63.5 & 32.4 & 4.1 & 42.1 & 0.92 & 3.45 \\
\hline 48 & 2141862 & 1387205 & 108020 & 62.9 & 31.4 & 3.7 & 98.0 & 64.1 & 32.0 & 3.9 & 42.2 & 0.9 & 3.3 \\
\hline
\end{tabular}

${ }^{\text {a }}$ 6, $N$-Hexadecyl- $N$-(2-methoxyethyl)- $N, N$-dimethylammonium bromide.

The mobile phase used for separation of products is 72/28- methanol/isopropanol.

${ }^{\mathrm{b}}$ HPLC calibration curve for $16-\mathrm{ArOH}$ : Area in $\mu \mathrm{V}=3.0 \times 10^{10}$ [16-ArOH] M - 28188 (5 points correlation coefficient $=0.99$ ); $\%$ yield of $16-$ $\mathrm{ArOH}=100 \times[16-\mathrm{ArOH}] \mathrm{M} /\left[16-\mathrm{ArN}_{2}^{+}\right] \mathrm{M}$.

HPLC calibration curve for $16-\mathrm{ArBr}$ : Area in $\mu \mathrm{V}=4.0 \times 10^{10}[16-\mathrm{ArBr}] \mathrm{M}-57195$ (5 points correlation coefficient $\left.=0.99\right)$; $\%$ yield of $16-\mathrm{ArBr}$ $=100 \times[16-\mathrm{ArBr}] \mathrm{M} /\left[16-\mathrm{ArN}_{2}^{+}\right] \mathrm{M}$.

HPLC calibration curve for 16-ArOHex: Area in $\mu \mathrm{V}=3.0 \times 10^{10}[16-\mathrm{ArOHex}] \mathrm{M}-19630$ (5 points correlation coefficient $=0.99$ ); $\%$ yield of 16 $\operatorname{ArOHex}=100 \times[16-\mathrm{ArOHex}] \mathrm{M} /\left[16-\mathrm{ArN}_{2}{ }^{+}\right] \mathrm{M}$. Observed yields are reproducible within $\pm 1 \%$ in duplicate experiments.

${ }^{\mathrm{c}}$ Because of quantitative conversions with no significant extraneous peak, observed yields were normalized and normalized yields were used to estimate the interfacial concentrations.

The correlation equation used between $\left[\mathrm{Br}_{\mathrm{i}}^{-}\right]$and $\% 16-\mathrm{ArBr} ;\left[\mathrm{HexOH}_{\mathrm{i}}\right]$ and $\% 16-\mathrm{ArOHex}$ are following:

$\% \quad \% 16-\mathrm{ArBr}=34.57\left[\mathrm{Br}_{\mathrm{i}}^{-}\right]^{0.65} \quad($ correlation coefficient $=1.00), \% 16-\mathrm{ArOHex}=1.214\left[\mathrm{HexOH}_{\mathrm{i}}\right]-0.1 \quad($ correlation coefficient $=0.99)$. 


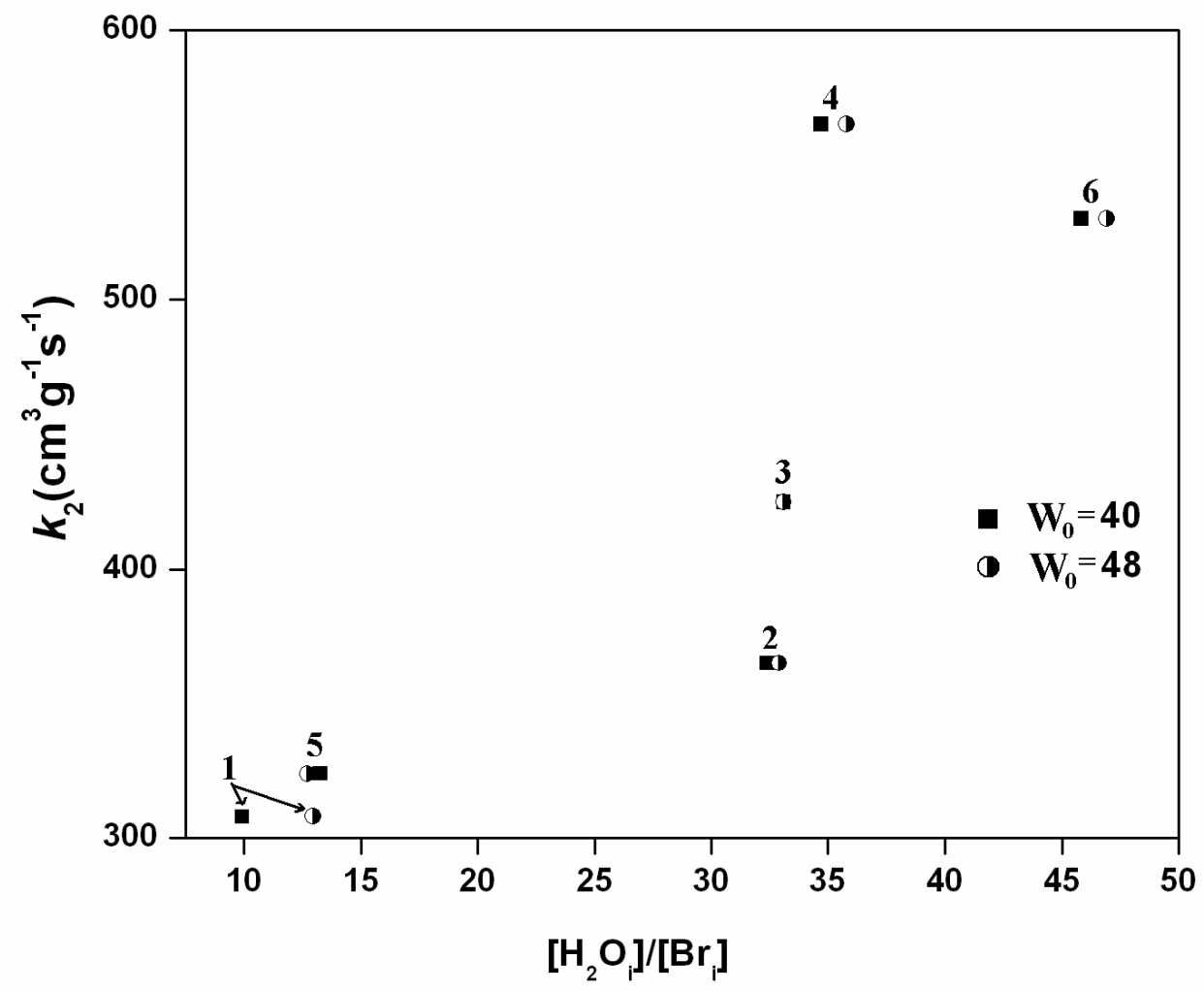

Figure S1. Variation of the second order rate constant $\left(k_{2}\right)$ for the lipase catalyzed hydrolysis of $p$-nitrophenyl- $n$-hexanoate with the ratio $\left[\mathrm{H}_{2} \mathrm{O}_{\mathrm{i}}\right] /\left[\mathrm{Br}_{\mathrm{i}}\right]$ in cationic $\mathrm{W} / \mathrm{O}$ microemulsions of 1-6 (at $\mathrm{W}_{0} 40$ and 48) formed at $z=4.8,25^{\circ} \mathrm{C}$ and $\mathrm{pH}=6.0(20 \mathrm{mM}$ phosphate). [Surfactant $]=50 \mathrm{mM}$, [Enzyme $]=1.02 \times 10^{-6} \mathrm{gmL}^{-1}$, [Substrate $]=3 \mathrm{mM}$ 